\title{
Relationship between Enhanced Positronium Formation and Enhanced Positron Trapping in Polymers at Low Temperature
}

\author{
J. KANSY ${ }^{a, *}$ AND T. SUZUKI ${ }^{b}$ \\ ${ }^{a}$ Institute of Material Science, Silesian University \\ Bankowa 12, 40-007 Katowice, Poland \\ ${ }^{b}$ High Energy Accelerator Research Organization (KEK) \\ 1-1 Oho, Tsukuba, Ibaraki 305-0801, Japan
}

\begin{abstract}
Positron annihilation lifetime spectroscopy is used for investigation of low-density polyethylene and ethylene-methyl methacrylate copolymers of $1.45,3.0$, and 5.4 mole $\%$ of methyl methacrylate. The lifetime spectra are collected at $30 \mathrm{~K}$, one by one, as a function of elapsed time. In the computer analysis a new theoretical model is developed, which enables separating the annihilation from positron free state, its trapped state and bound state in positronium. The positron trapping rate $\mu$ and the enhanced positronium formation rate $\kappa$ are determined. The calculated values of $\mu$ and $\kappa$ turned out to be linearly correlated. This correlation presumably originates from an influence of trapped electrons on the trapping of positrons. The dependences of $\kappa$ on measurement time are determined for low-density polyethylene and ethylene-methyl methacrylate of different methyl methacrylate content. A theoretical model describing quantitatively the dependences is proposed. The model considers the processes of electron-ion recombination, electron trapping, and electron scavenging by dipolar carbonyl groups supplied by methyl methacrylate additives.
\end{abstract}

PACS numbers: 78.70.Bj, 71.60.+z, 61.80.Fe

\section{Introduction}

It is well known that positron injected into molecular substances after short spur/blob reactions [1-3] can bind an electron and form a positronium (Ps) in a short-lived singlet state ( ara-Ps, $p$-Ps) or in a long-lived triplet state (ortho-Ps, $o$-Ps). The statistical ratio of the formation probability of $p$-Ps to o-Ps is 1:3.

*corresponding author; e-mail: kansy@us.edu.pl 
Recently the enhanced positronium formation was observed and it was explained by positron reaction with weakly bound electrons stored in polymer at low temperatures [4-6]. It was suggested [7] that a delayed formation of Ps by a freely diffusing positron and an electron bound in a shallow potential was also possible. In our previous paper [8] we proposed a model which linked the enhancement of Ps production with the delayed formation of Ps. The model was used for fitting a series of positron annihilation lifetime (PAL) spectra for low-density polyethylene (LDPE) and high-density polyethylene (HDPE). Obtained results suggested that the diffusing positrons, besides forming Ps, could also be trapped by active centers in polymer structure. Moreover, this positron trapping turned out to be correlated with the delayed formation of Ps.

The purpose of this work is to check validity of the model, introduced in our previous paper, for description of PAL spectra for LDPE and ethylene-methyl methacrylate (EMMA) measured at low temperature. Recently the materials have been investigated intensively [9-11]. It was shown that carbonyl groups introduced into polyethylene matrix by MMA additives strongly inhibit Ps formation due to scavenging of electrons and positrons [11]. We hope that our model is able to quantitatively describe those processes in order to understand better the mechanisms influencing the positronium formation in polymers.

\section{Theoretical model of the PAL spectrum}

The theoretical model used in the present analysis of PAL spectra is based on the following assumptions. A positron injected into polymer takes part in fast processes inside the positron spur and then leaves the terminal spur (blob) as a free particle or as a loosely bound pair $\mathrm{e}^{+}-\mathrm{e}^{-}$(quasi-positronium, q-Ps) [3]. Because of the spin exchange repulsion between the electron of the pair $\mathrm{e}^{+}-\mathrm{e}^{-}$and the core electrons, the q-Ps is pushed out, into the free spaces of larger and larger size. In this way, excitation of the pair becomes lower, providing a gradual transformation of q-Ps into a stable Ps and its final localization in the elementary free volume. The localization process is slow because an immediate transfer of large amount of energy to the heat bath is forbidden. We called the processes described above [8] the slow localization of positronium (SLP).

If a free positron leaves the blob, it diffuses through the material covering a relatively long distance (the estimated diffusion length in polyethylene is estimated to be $80 \mathrm{~nm}$ [12]). It annihilates from the free or trapped states or forms a positronium after meeting a weakly trapped electron. In the last case, the positronium is formed and localized almost instantaneously because, contrary to SLP, the great amount of Ps binding energy is lost for picking up the electron from the trap. We called this process the delayed formation of positronium (DFP).

\subsection{Slow localization of positronium}

We describe the change of the annihilation rate $\lambda_{o / p}(t)$ of both $o$ - and $p$-Ps during SLP by an empirical relation which takes into account the gradual internal 
relaxation and the slow localization of Ps

$$
\lambda_{o / p}(t)=\lambda_{o / p \text { intr }}(t)+\lambda_{p-o}(t)
$$

where

$$
\lambda_{o / p \operatorname{intr}}(t)=\lambda_{o / p \text { intr }}(\infty)\left[1-\exp \left(-t / \tau_{\text {relax }}\right)\right]
$$

describes the increase in the intrinsic annihilation rate of q-Ps due to its internal relaxation, and the relation

$$
\lambda_{p-o}(t)=\lambda(\infty)+[\lambda(0)-\lambda(\infty)] \exp \left(-t / \tau_{\text {local }}\right)
$$

approximates the gradual decrease in the Ps pick-off annihilation rate caused by the slow localization of Ps; $\lambda_{o / p}$ intr $(\infty)$ is the intrinsic annihilation rate of the stable $o-/ p$-Ps; $\lambda(\infty)$ denotes the asymptotic (after Ps localization in the free volume) pick-off lifetime, whereas $\lambda(0)$ is the initial pick-off lifetime of q-Ps. We assume that $\lambda(0)$ is equal to $\lambda_{+}$- the free positron annihilation rate; $\tau_{\text {relax }}$ represents the average time of transformation of q-Ps into the stable Ps and $\tau_{\text {local }}$ is the average time of Ps localization.

The part of PAL spectrum representing the $o$ - $/ p$-Ps component $\left(C_{o / p, \mathrm{Ps}}\right)$ due to SLP is given by an integral. This integral can be calculated analytically as a series of exponential functions

$$
C_{o / p, \operatorname{Ps}}=\lambda_{o / p}(t) \exp \left[-\int_{0}^{t} \lambda_{o / p}(t) \mathrm{d} t\right]=\sum_{k, j=0}^{\infty} \frac{I_{k j}}{\tau_{k j}} \exp \left(-\frac{t}{\tau_{k j}}\right),
$$

where

$$
I_{k j}=\frac{s^{k}}{k !} \frac{r^{j}}{j !} \exp (-s-r), \quad s=-\lambda_{o / p} \operatorname{intr}(\infty) \tau_{\text {relax }}, \quad r=[\lambda(0)-\lambda(\infty)] \tau_{\text {local }}
$$

and

$$
\tau_{k j}^{-1}=\left[\lambda_{o / p \operatorname{intr}}(\infty)+\lambda(\infty)\right]+k / \tau_{\text {relax }}+j / \tau_{\text {local }} .
$$

\subsection{Free positron trapping and DFP process}

The "free" positron diffuses through the material and (1) annihilates from its unbounded state with the annihilation rate $\lambda_{+}$, or (2) becomes captured by a trapping center with the rate $\mu$ and annihilates in this state with the rate $\lambda_{+}^{\mathrm{t}}$, or (3) together with a weakly trapped electron forms Ps with the rate $\kappa$. As it was already mentioned, we believe that Ps in DFP is formed in its stable and localized state almost immediately; therefore, just after $o-/ p$-Ps formation, the annihilation rate is $\lambda_{o / p}=\lambda_{o / p} \operatorname{intr}(\infty)+\lambda(\infty)$. The spectrum components for free $\mathrm{e}^{+}$, trapped $\mathrm{e}^{+}, p$-Ps and $o$-Ps originated from DFP are

$$
B_{+}=\lambda_{+} c_{+}, \quad B_{+}^{\mathrm{t}}=\lambda_{+}^{\mathrm{t}} c_{+}^{\mathrm{t}}, \quad B_{p}=\lambda_{p} c_{p}, \quad \text { and } B_{o}=\lambda_{o} c_{o},
$$

where $c_{+}, c_{+}^{\mathrm{t}}, c_{p}$ and $c_{o}$ denote the probabilities to find a free $\mathrm{e}^{+}$, a trapped $\mathrm{e}^{+}$, $p$-Ps and $o$-Ps at instant $t$, whereas the lambdas are respective annihilation rates in each of these processes. The probabilities can be calculated from a system of kinetic equations. Their solutions are [8]: 


$$
\begin{aligned}
c_{+} & =c_{0+} \exp (-\lambda t), \\
c_{+}^{\mathrm{t}} & =\frac{c_{0+} \mu}{\lambda-\lambda_{+}^{\mathrm{t}}}\left[\exp \left(-\lambda_{+}^{\mathrm{t}} t\right)-\exp (-\lambda t)\right], \\
c_{p} & =\frac{\frac{1}{4} c_{0+} \kappa}{\lambda-\lambda_{p}}\left[\exp \left(-\lambda_{p} t\right)-\exp (-\lambda t)\right], \\
c_{o} & =\frac{\frac{3}{4} c_{0+} \kappa}{\lambda-\lambda_{o}}\left[\exp \left(-\lambda_{o} t\right)-\exp (-\lambda t)\right],
\end{aligned}
$$

where $\lambda=\lambda_{+}+\mu+\kappa$ and $c_{0+}=c_{+}(0)$.

The full shape of PAL spectrum (without consideration the background and the resolution) is (Eqs. (4), (5))

$$
F(t)=I_{\mathrm{blob}}\left(\frac{1}{4} C_{p, \mathrm{Ps}}+\frac{3}{4} C_{o, \mathrm{Ps}}\right)+\left(1-I_{\mathrm{blob}}\right)\left(B_{+}+B_{+}^{\mathrm{t}}+B_{o}+B_{p}\right) .
$$

The model contains eight parameters which have to be determined by its fitting to experimental data. These are: $I_{\text {blob }}$ (the efficiency of Ps production in the blob), $\lambda_{+}=\lambda(0) \equiv \tau^{-1}(0), \lambda(\infty) \equiv \tau^{-1}(\infty), \lambda_{+}^{\mathrm{t}} \equiv 1 / \tau_{+}^{\mathrm{t}}, \tau_{\text {relax }}, \tau_{\text {local }}, \mu$ and $\kappa$.

Function $F(t)$ can be divided into two parts

$$
F(t)=f(t)+I \lambda_{o} \exp \left(-\lambda_{o} t\right)
$$

where

$$
I=\frac{3}{4} I_{\mathrm{blob}} \exp (-s-r)+\left(1-I_{\mathrm{blob}}\right) \frac{\frac{3}{4} c_{0+} \kappa}{\lambda-\lambda_{o}}
$$

and $f(t)$ is the short-lived part of $F(t)$. The second term in Eq. (11) is an exponential function with the time constant equal to the $o$-Ps lifetime. This term, in the 3-discrete-component model of PAL spectrum, is considered as the third component related to the $o$-Ps contribution. In this case, the coefficient $I$ plays role of $I_{3}$ - the intensity of the third component. Therefore, taking into account the explicit expressions for $s$ and $r$ (Eq. (4)), one can conclude that

$$
I_{3} \cong \frac{3}{4} I_{\text {blob }} \exp \left\{-[\lambda(0)-\lambda(\infty)] \tau_{\text {local }}\right\}+\left(1-I_{\text {blob }}\right) \frac{\frac{3}{4} c_{0+} \kappa}{\lambda-\lambda_{o}} .
$$

(In the exponential function we neglected a small term $s=\tau_{\text {relax }} / 142$.)

The first term in Eq. (13) relates to the efficiency of $o$-Ps formation in the blob, whereas the second one represents the enhancement of $o$-Ps formation due to DFP.

\section{Experimental and analysis}

In the studies, low-density polyethylene (LDPE G201, crystallinity $55.3 \%$, Sumitomo Chemical Co., Ltd.) and ethylene-methyl methacrylate copolymers (1.45, 3.0 and 5.4 mole\% of MMA) were used. Lifetime spectra were measured with a "fast-fast" positron lifetime spectrometer. The activity of positron source, ${ }^{22} \mathrm{Na}$, was about 3.7 MBq. The sample-source-sample in sandwich geometry was fixed on the cooling finger of a helium cryostat (CW303, Iwatani Plantech Co.). 
Each of the samples was cooled down from room temperature to $30 \mathrm{~K}$ in about $1 \mathrm{~h}$. Then the PAL spectra were measured at $30 \mathrm{~K}$ in darkness as a function of elapsed time. Each PAL spectrum was collected within 1 hour, which resulted in statistics of about 1 million counts. One sample (EMMA with $3 \%$ MMA) at $123 \mathrm{~h}$ after cooling was lighted by a source of visible light and the spectra were collected for next 4 hours.

The experimental data were analyzed with LT [13] v.10 program. The theoretical model, described in the previous section, was directly included into the code of the program. Each series of spectra (i.e. all the spectra related to the same sample but measured at different hours of elapsed time) were analyzed together (i.e. during the same fitting process). Some of the model parameters (see next section) as well as the parameters for source correction and parameters related to the resolution curve had the same values for all spectra of a given series. The "source parameters" were fixed at $10 \%$ for source contribution and 0.386 ns for annihilation lifetime in the source material. The parameters related to the resolution curve (2 shifted Gaussians) were fitting parameters but their values were accepted to be the same for all spectra of the series.

\section{Results}

The quality of the fits of the theoretical model to the experimental data was very good. The mean value of the fitting variances was 1.06.

Table I shows the determined values of fitting parameters, which were assumed (or turned out to be) independent of the elapsed time of measurement $\left(t_{\mathrm{m}}\right)$. Particularly, we assumed that $I_{\mathrm{blob}}$, i.e. the total fraction of positronium (both ortho and para) which has been produced due to the blob reaction, did not depend on $t_{\mathrm{m}}$.

TABLE I

The parameters independent of the measurement time determined from analysis of the PAL spectra (measured at $30 \mathrm{~K}$ ) for the investigated samples.

\begin{tabular}{|c|c|c|c|c|c|c|}
\hline & $I_{\text {blob }}[\%]$ & $\tau(\infty)[\mathrm{ns}]$ & $\tau(0)[\mathrm{ns}]$ & $\tau_{+}^{\mathrm{t}}[\mathrm{ns}]$ & $\tau_{\text {relax }}[\mathrm{ns}]$ & $\tau_{\text {local }}[\mathrm{ns}]$ \\
\hline LDPE & 48.8 & 1.32 & 0.334 & 0.450 & \multirow{4}{*}{0.076} & \multirow{4}{*}{0.121} \\
\hline EMMA (1.45\% MMA) & 31.2 & 1.32 & \multirow{3}{*}{0.317} & \multirow{3}{*}{0.378} & & \\
\hline EMMA (3\% MMA) & 30.3 & 1.34 & & & & \\
\hline EMMA (5.4\% MMA) & 28.6 & 1.34 & & & & \\
\hline
\end{tabular}

The asymptotic pick-off lifetime of Ps $\tau(\infty)$ (the equivalent of $\tau_{3}$ in the three-component conventional analysis) was treated as an entirely free parameter. In spite of this, it turned out that for a given series of spectra values of $\tau(\infty)$ were almost constant. They only fluctuated a bit in the range of $\pm 0.01 \mathrm{~ns}$. Table I contains averaged values of $\tau(\infty)$ for particular samples. 
During analysis of the spectra for LDPE, each of the following parameters (besides $\left.I_{\text {blob }}\right)$ had a free but common value for the whole series: $\tau(0)$ - the initial pick-off lifetime of q-Ps, $\tau_{+}^{\mathrm{t}}$ - the lifetime of a trapped positron, $\tau_{\text {relax }}-$ the positronium internal relaxation time, and $\tau_{\text {local }}$ - the Ps localization time.

In the case of the fitting of the spectra for EMMA, both $\tau_{\text {relax }}$ and $\tau_{\text {local }}$ were fixed at values obtained for LDPE. Additionally, $\tau(0)$ and $\tau_{+}^{\mathrm{t}}$ were also fixed but at the values different from those for $\mathrm{LDPE}^{\dagger}$. Because of possible different nature of $\mathrm{e}^{+}$traps $\tau_{+}^{\mathrm{t}}$ can have many values or can be even distributed. However, in our analysis, to avoid introducing too many free parameters, we assumed only one (average) value of $\tau_{+}^{\mathrm{t}}$ for LDPE and another value of $\tau_{+}^{\mathrm{t}}$ for all samples of EMMA, independently of MMA content.

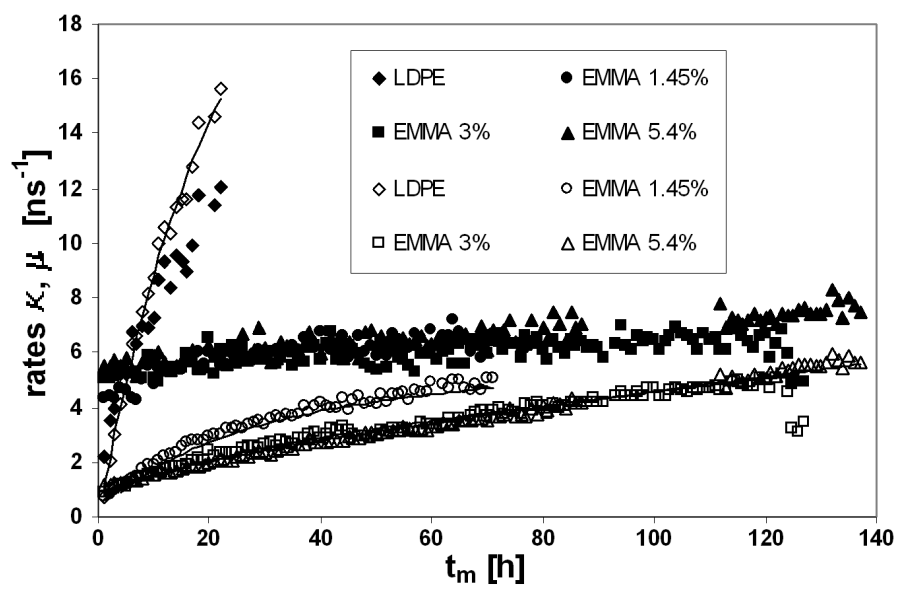

Fig. 1. The positron trapping rate $\mu$ (solid symbols) and the delayed Ps formation rate $\kappa$ (open symbols) versus the measurement time $t_{\mathrm{m}}$ for LDPE and EMMA copolymers of different MMA content. Almost all points were measured in dark with the exception of the four last points on the EMMA ( $3 \%$ MMA) curve. The solid lines at $\kappa$-points represent theoretical values fitted according to the model described in Appendix. In the fitting we ignored the points related to the measurement in light.

Figure 1 shows the $\mathrm{e}^{+}$trapping rate $\mu$ (solid symbols) and the delayed Ps formation rate $\kappa$ (open symbols) as a function of measurement time $t_{\mathrm{m}}$ for LDPE and the investigated EMMA copolymers. Almost all points were measured in dark. It is seen that in dark $\kappa$ approaches higher and higher value with elapsing time $t_{\mathrm{m}}$. However, the increase in $\kappa$ is strongly suppressed even by a small amount of MMA in the sample. A few last points related to EMMA of $3 \%$ MMA were measured in light. Rapid reduction of $\mu$ and $\kappa$ after illumination of the sample is also remarkable.

\footnotetext{
${ }^{\dagger}$ Values of these parameters were established by averaging the results obtained in a preliminary analysis.
} 


\section{Discussion}

As it is known from literature, the positronium formation in polymers is affected by presence of shallow electron traps in the polymer structure [4-7], electron or/and positron scavengers, such as carbonyl groups of dipolar character [9-11], free radicals generated by irradiation [14-16], etc.

The efficiencies of Ps formation inside the blob $I_{\text {blob }}$ for the investigated samples are shown in Table I. The determined values of $I_{\text {blob }}$ are higher than $\frac{4}{3} I_{3}\left(t_{\mathrm{m}}=0\right)$ [11] (i.e. the $o$-Ps intensity obtained in the conventional 3-component analysis at the beginning of measurements). This result can be understood on the basis of Eq. (13). Providing $\kappa \cong 0$ at $t_{\mathrm{m}}=0$ (Fig. 1) Eq. (13) can be simplified

$\frac{4}{3} I_{3}\left(t_{\mathrm{m}}=0\right)=I_{\text {blob }} \exp \left\{-\left[\tau^{-1}(0)-\tau^{-1}(\infty)\right] \tau_{\text {local }}\right\}$.

After inserting the explicit values of parameters from Table I into Eq. (14), $\frac{4}{3} I_{3}\left(t_{\mathrm{m}}=0\right) / I_{\text {blob }}$ can be estimated. Because of similar values of $\tau(0), \tau(\infty)$, and common value of $\tau_{\text {local }}$, the ratio $\frac{4}{3} I_{3}\left(t_{\mathrm{m}}=0\right) / I_{\text {blob }}$ is about 0.75 for LDPE and all of the EMMA samples. It is obvious that $\frac{4}{3} I_{3}\left(t_{\mathrm{m}}=0\right)$ and $I_{\mathrm{blob}}$ would be equal if Ps formed in the blob were localized immediately (i.e. $\tau_{\text {local }}=0$ ).

One observes a distinct jump of $I_{\text {blob }}$ from high value for LDPE to lower values for the EMMA samples. This seems to confirm an effective scavenging of positrons and electrons by MMA additives [10]. $I_{\text {blob }}$ descends slightly with the increasing content of MMA. It suggests that the scavenging is almost saturated, even for the EMMA copolymer with $\approx 1$ mole\% MMA.

\subsection{Enhanced Ps formation in EMMA copolymers}

To explain the dependences of $\kappa$ on $t_{\mathrm{m}}$ shown in Fig. 1, we assume that there are two types of electron traps in EMMA copolymers. Shallow traps ${ }^{\ddagger}(\mathrm{ST})$ are distributed in ethylene matrix, and deeper traps, connected with the carbonyl groups (CG), are introduced by MMA additives. We suppose that the concentration of electron traps for whole $t_{\mathrm{m}}$ is constant ${ }^{\S}$. The excess electrons generated by the source are captured by both kinds of traps with different efficiency. Additionally, we assume that the trapped electrons can be released from ST with the rate of $\beta_{t}$, whereas the complexes $e^{+}-C G$ are stable. Some of the captured electrons can be picked up by freely diffusing positrons to form positronium. A quantitative model of the processes is described in Appendix. The model has been fitted to the experimental values of $\kappa\left(t_{\mathrm{m}}\right)$. The solid lines in Fig. 1 represent the fitted values of $\kappa$. The determined values of the fitting parameters are shown in Table II. The trapping rate, $\nu_{\mathrm{M}}$, by CG (extrapolated to $100 \%$ of poly(methyl methacrylate)

${ }^{\ddagger}$ The nature of these traps is not well explained in literature. There are suggestions that they are free radicals, free volume centers, active chemical groups produced by chemical reactions of free radicals and some admixtures included in polymer, etc.

${ }^{\S}$ In spite of the fact that some amount of electron traps can be created by the positron source irradiation, because of relatively low activity of the source, we believe that the amount of generated $\mathrm{e}^{+} / \mathrm{e}^{-}$traps can be neglected. 
(PMMA)) is found to be about 400 times higher than $\nu_{\mathrm{t}}$, i.e. the trapping rate by ST (in $100 \%$ of LDPE). The number density $\left(n_{\mathrm{M}}\right)$ of electrons in CG is about two orders of magnitude higher than the number density $\left(n_{\mathrm{t}}\right)$ in ST for EMMA samples after long enough $t_{\mathrm{m}}$ (Fig. 2). The absolute values of $n_{\mathrm{M}}$ and $n_{\mathrm{t}}$ could not be determined directly from our calculations (we could determine $n_{\mathrm{M}} / n_{\mathrm{t}}$ only). Therefore the absolute values were estimated on the assumption that the production of free electrons, $\alpha$, by the positron source irradiation was $5 \times 10^{14} \mathrm{~g}^{-1} \mathrm{~s}^{-1}$. The predominant trapping of $\mathrm{e}^{+}$by $\mathrm{CG}$ compared to $\mathrm{ST}$ can explain different values of $\lambda_{+}^{\mathrm{t}}$ for the EMMA and LDPE samples.

TABLE II

Fitted values of parameters of the theoretical model described in Appendix.

\begin{tabular}{c|c|c|c|c}
\hline \hline$\alpha \kappa_{0 \mathrm{t}}\left[\mathrm{s}^{-1} \mathrm{~g}^{-1} \mathrm{~ns}^{-1}\right]$ & $\nu_{\mathrm{M}} / \nu_{\mathrm{t}}$ & $\nu_{\mathrm{R}} / \kappa_{0 \mathrm{t}} \mu_{\mathrm{t}}$ & $\beta_{\mathrm{t}}\left[\mathrm{h}^{-1}\right]$ & $\kappa_{0 \mathrm{M}} / \kappa_{0 \mathrm{t}}$ \\
\hline $9.77 \times 10^{-3}$ & 429 & 31.4 & 0.059 & 0.031
\end{tabular}

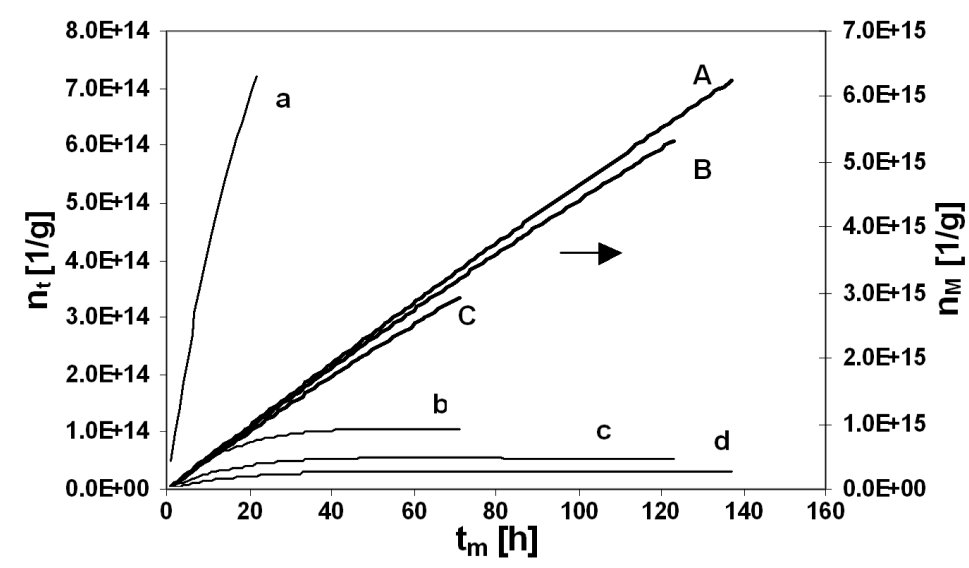

Fig. 2. Number density of electrons trapped in shallow traps versus $t_{\mathrm{m}}$ for: $a-\mathrm{LDPE}$, $b-\operatorname{EMMA}(1.45 \% \mathrm{MMA}), c-\operatorname{EMMA}(3 \% \mathrm{MMA}), d-\operatorname{EMMA}(5.4 \% \mathrm{MMA})$ and $t_{\mathrm{m}}$ - dependence of number density of electrons attached to carbonyl groups (right axis) for: $A-\operatorname{EMMA}(1.45 \% \mathrm{MMA}), B-\operatorname{EMMA}(3 \% \mathrm{MMA}), C-\operatorname{EMMA}(5.4 \% \mathrm{MMA})$. The quantities were calculated on the basis of a theoretical model (Appendix). The absolute values of the number densities depend on the positron source activity. In this calculation we assumed that the source produced per second $5 \times 10^{14}$ free electrons/gram of sample.

We assumed that in the EMMA samples the diffusing positron could form Ps with an electron picked up from ST as well as from a trap generated by CG. The obtained value of $\kappa_{0 \mathrm{M}} / \kappa_{0 \mathrm{t}}$, i.e. the ratio of the specific rate of the delayed Ps formation of electrons in CG to that in ST, is only 0.03. However, for enough 
long time $t_{\mathrm{m}}$, because of the high values of $n_{\mathrm{M}}$ (Fig. 2), the amount of positronium formed with the electrons picked up from the CG traps becomes significant.

\subsection{A correlation between delayed formation of positronium and efficiency of positron trapping}

In our previous paper [8] we stated that in HDPE and LDPE samples the Ps formation rate $(\kappa)$ is linearly correlated with the rate of positron trapping $(\mu)$. Moreover, after illumination of the HDPE sample, both $\kappa$ and $\mu$ decreased to their initial values at $t_{\mathrm{m}}=0$. This statement is fully confirmed for the examined samples. Figure 3 shows plots $\mu$ on $\kappa$ for these samples. Respective points

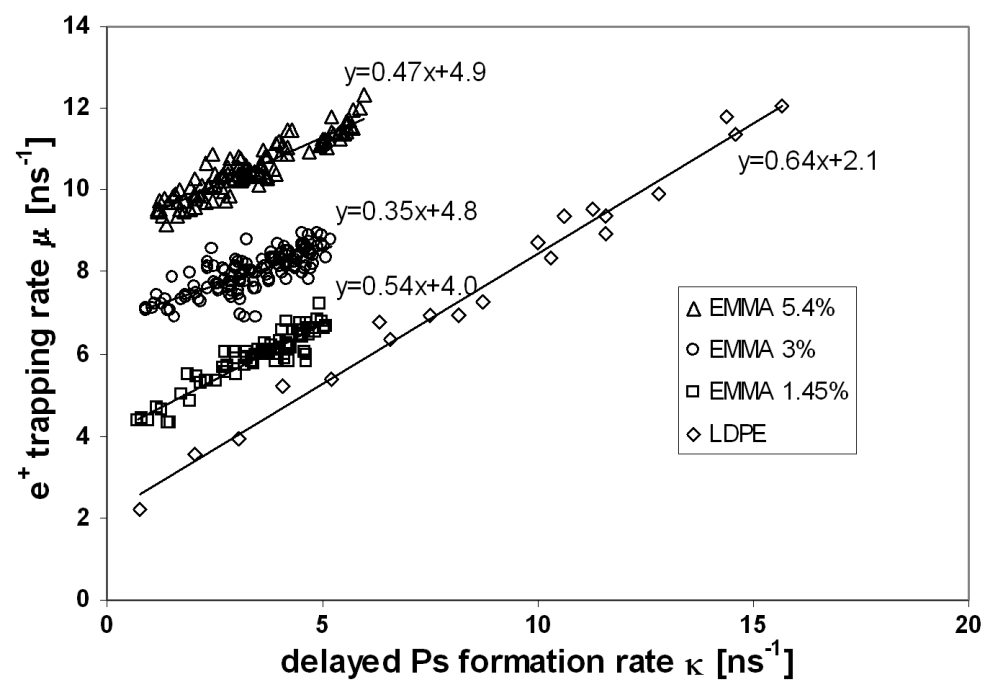

Fig. 3. Plots $\mu$ versus $\kappa$ (shown in Fig. 1) related to LDPE and EMMA copolymers of different MMA content. For seeing conveniently, the points related to EMMA (5.4\% MMA) and EMMA (3\% MMA) are shifted up by 4 and 2 units, respectively. The solid lines represent the linear correlations of $\mu$ and $\kappa$.

are fitted well with straight lines. Value of $\mu$ at $t_{\mathrm{m}} \approx 0$ can be interpreted as the efficiency of positron trapping in absence of the trapped electrons in polymer, whereas increase in $\mu$ for $t_{\mathrm{m}}>0$ represents an enhancement of $e^{+}$trapping at higher number densities of the trapped electrons. It is seen that values of $\mu$ at $t_{\mathrm{m}} \approx 0$ determined for the EMMA copolymers decrease moderately with the increase in the MMA content. In pure LDPE $\mu$ at $t_{\mathrm{m}} \approx 0$ is significantly lower. The observed correlation between the enhancement of $\mu$ and $\kappa$ suggests that the positron trapping and the DFP process are related. A possible explanation of this interconnection can be as follows. At $t_{\mathrm{m}} \approx 0$ the positron diffuses through the material entirely randomly. However, for $t_{\mathrm{m}} \gg 0$ the number of trapped electrons increases, and the probability of meeting such an electron by the "free" positron becomes higher and higher. If the positron accidentally finds 
itself within the interaction range of any of the trapped electrons, it moves directly to this electron. The positron coming closer to the electron can eventually be scavenged by the dipole trap. The bleaching of electrons by visible light makes the $\mathrm{e}^{+}$-diffusion random again, which radically lowers $\mu$ to its initial value as it could be observed for the EMMA (3\% MMA) sample illuminated at $73 \mathrm{~h}$ (Fig. 1) and for HDPE in our previous work [8].

\section{Conclusions}

The experimental data related to the examined samples of LDPE and EMMA copolymers can be described very well by the new theoretical model of PAL spectra for polymers at low temperatures. We consider the perfect fits and the coherence of obtained results as strong arguments in favor of validity of the model. The shape of the PAL spectrum is connected directly with the processes in which positron is involved that enables to use quantities of well defined physical meanings as the fitting parameters of the model. Moreover, the special fitting procedure, which allows to use the same set of the fitting parameters to a large number of lifetime spectra, ensures high reliability of the obtained results.

In the investigated samples, the enhancement of positronium formation as well as the enhancement of positron trapping rate along the measurement time are observed. A strong correlation between the enhancement of positronium formation and the enhancement of positron trapping rate suggests that both processes are related. The enhancement of Ps formation can be fully explained by the processes of electron-ion recombination, electron trapping, and electron scavenging.

\section{Appendix. Mechanism of enhanced positronium formation in EMMA at low temperature}

Let $\alpha$ be the number density of free electrons generated in a unit of time by the radiation of the positron source. The electrons recombine with positive ions or they are captured by some shallow traps existing in the sample. Additionally, in the EMMA samples the electrons are scavenged by carbonyl groups supplied by the MMA additives. The number density $\left(n_{\mathrm{t}}\right)$ of trapped electrons in ST increases with the rate $r_{1}=\alpha c_{\mathrm{E}} \nu_{\mathrm{t}} / D$. The denominator $D=c_{\mathrm{E}} \nu_{\mathrm{t}}+c_{\mathrm{M}} \nu_{\mathrm{M}}+n_{\text {ion }} \mu_{\mathrm{R}}$ takes into account the three mentioned processes in which the free electrons are involved: $c_{\mathrm{E}} \nu_{\mathrm{t}}$ is the trapping rate into $\mathrm{ST}, c_{\mathrm{M}} \nu_{\mathrm{M}}$ is the rate of electron scavenging by $\mathrm{CG}$ in MMA and $n_{\text {ion }} \mu_{\mathrm{R}}$ is the electron-ion recombination rate, where $c_{\mathrm{M}}$ denotes the mole concentration of MMA, $c_{\mathrm{E}}=1-c_{\mathrm{M}}, \mu_{\mathrm{R}}$ is the electron-ion recombination specific rate, and $n_{\text {ion }}$ is the number density of positive ions. We assume that $n_{\text {ion }}=n_{\mathrm{t}}+n_{\mathrm{M}}$, where $n_{\mathrm{t}}$ is the number density of trapped electrons in ST and $n_{\mathrm{M}}$ is the number density of electrons bound by CG. Because of thermal activations the trapped electrons can be released from ST with the rate $r_{2}=\beta_{\mathrm{t}} n_{\mathrm{t}}$. Further on, they can recombine with positive ions or be scavenged by CG or be re-trapped by ST with the efficiency $r_{3}=\beta_{\mathrm{t}} n_{\mathrm{t}} c_{\mathrm{E}} \nu_{\mathrm{t}} / D$. Therefore, the total change of the number 
density of electrons inside $\mathrm{ST}$ is $\mathrm{d} n_{\mathrm{t}} / \mathrm{d} t_{\mathrm{m}}=r_{1}-r_{2}+r_{3}$. After substitution the explicit terms and simple transformations

$$
\mathrm{d} n_{\mathrm{t}} / \mathrm{d} t_{\mathrm{m}}=\left\{\alpha c_{\mathrm{E}} \nu_{\mathrm{t}}-\beta_{\mathrm{t}} n_{\mathrm{t}}\left[c_{\mathrm{M}} \nu_{\mathrm{M}}+\left(n_{\mathrm{t}}+n_{\mathrm{M}}\right) \mu_{\mathrm{R}}\right]\right\} / D .
$$

Taking into account the efficiency of capturing of electrons originating from the ionizations $\left(\alpha c_{\mathrm{M}} \nu_{\mathrm{M}} / D\right)$ and from the decayed $\mathrm{ST}\left(\beta_{\mathrm{t}} n_{\mathrm{t}} c_{\mathrm{M}} \nu_{\mathrm{M}} / D\right)$ the increase in the number of electrons joined to $\mathrm{CG}$ can be determined as

$$
\mathrm{d} n_{\mathrm{M}} / \mathrm{d} t_{\mathrm{m}}=\left(\alpha+\beta_{\mathrm{t}} n_{\mathrm{t}}\right) c_{\mathrm{M}} \nu_{\mathrm{M}} / D \text {. }
$$

It is known that the enhancement of positronium formation at low temperatures depends on the number of electrons trapped in ST. As a hypothesis we assume that the electrons attached to the carbonyl groups can also give a contribution to these enhancement

$$
\kappa=\kappa_{0 \mathrm{t}} n_{\mathrm{t}}+\kappa_{0 \mathrm{M}} n_{\mathrm{M}},
$$

where $\kappa$ is the rate of positronium formation in the DFP process, whereas $\kappa_{0 \mathrm{t}}$ and $\kappa_{0 \mathrm{M}}$ are the specific Ps formation rates from the electrons trapped in ST and in $\mathrm{CG}$, respectively.

After multiplying Eqs. (A1) and (A2) by $\kappa_{0 \mathrm{t}}$ and simple transformations, the following system of differential equations is obtained:

$$
\begin{aligned}
\frac{\mathrm{d} \kappa^{\prime}}{\mathrm{d} t_{\mathrm{m}}} & =\frac{c_{\mathrm{E}} p_{1}-\beta_{\mathrm{t}} \kappa^{\prime}\left[c_{\mathrm{M}} p_{2}+\left(\kappa^{\prime}+\kappa^{\prime \prime}\right) p_{3}\right]}{c_{\mathrm{E}}+c_{\mathrm{M}} p_{2}+\left(\kappa^{\prime}+\kappa^{\prime \prime}\right) p_{3}}, \\
\frac{\mathrm{d} \kappa^{\prime \prime}}{\mathrm{d} t_{\mathrm{m}}} & =\frac{\left(p_{1}+\beta_{\mathrm{t}} \kappa^{\prime}\right) c_{\mathrm{M}} p_{2}}{c_{\mathrm{E}}+c_{\mathrm{M}} p_{2}+\left(\kappa^{\prime}+\kappa^{\prime \prime}\right) p_{3}},
\end{aligned}
$$

where $\kappa^{\prime}=\kappa_{0 \mathrm{t}} n_{\mathrm{t}}$ and $\kappa^{\prime \prime}=\kappa_{0 \mathrm{t}} n_{\mathrm{M}}$. The system contains four unknown parameters: $p_{1}=\alpha \kappa_{0 \mathrm{t}}, p_{2}=\nu_{\mathrm{M}} / \nu_{\mathrm{t}}, p_{3}=\mu_{\mathrm{R}} / \kappa_{0 \mathrm{t}} \nu_{\mathrm{t}}$ and $p_{4}=\beta_{\mathrm{t}}$. The parameters were determined by fitting Eq. (A3) to the experimental values of $\kappa$. The fitting was performed simultaneously to all of the experimental curves $\kappa\left(t_{\mathrm{m}}\right)$ related to samples of different content of MMA (Fig. 1). During the fitting procedure, the system of differential equations (Eqs. (A4), (A5)) was solved numerically for current values of fitting parameters. The total number of fitting parameters was five, i.e. $p_{1}$ to $p_{4}$ and $p_{5}=\kappa_{0 \mathrm{M}} / \kappa_{0 \mathrm{t}}$.

\section{Acknowledgments}

The authors are grateful to Professor V. Shantarovich for variable discussions and comments on this manuscript.

\section{References}

[1] O.E. Mogensen, J. Chem. Phys. 60, 998 (1974).

[2] O.E. Mogensen, Positron Annihilation in Chemistry, Springer-Verlag, Berlin 1995.

[3] S.V. Stepanov, V.M. Byakov, J. Chem. Phys. 116, 6178 (2002).

[4] C.L. Wang, T. Hirade, F.H.J. Maurer, M. Eldrup, N.J. Pedersen, J. Chem. Phys. 108, 4654 (1998). 
[5] T. Hirade, F.H.J. Maurer, M. Eldrup, Radiat. Phys. Chem. 58, 465 (2000).

[6] Y. Ito, T. Hirade, E. Hamada, T. Suzuki, Y. Ito, Acta Phys. Pol. A 95, 533 (1999).

[7] N. Suzuki, T. Hirade, F. Saito, T. Hyodo, Radiat. Phys. Chem. 68, 647 (2003).

[8] J. Kansy, T. Suzuki, Radiat. Phys. Chem., in press.

[9] T. Suzuki, C. He, V.P. Shantarovich, K. Kondo, E. Hamada, M. Matsuo, L. Ma, Y. Ito, Radiat. Phys. Chem. 66, 161 (2003).

[10] V.P. Shantarovich, T. Suzuki, C. He, V.W. Gustov, Radiat. Phys. Chem. 67, 15 (2003).

[11] C. He, V.P. Shantarovich, T. Suzuki, S.V. Stepanov, R. Suzuki, M. Matsuo, J. Chem. Phys. 122, 214907 (2005).

[12] R.S. Bursa, M. Duarte Naia, D. Margoni, A. Zecca, Appl. Phys. A 60, 447 (1995).

[13] J. Kansy, Nucl. Instrum. Methods Phys. Res. A 374, 235 (1996).

[14] T. Suzuki, Y. Ito, K. Kondo, E. Hamada, Y. Ito, Radiat. Phys. Chem. 58, 485 (2000).

[15] T. Suzuki, Y. Ito, K. Kondo, E. Hamada, Z.Q. Chen, Y. Ito, Radiat. Phys. Chem. 60, 535 (2001).

[16] R.S. Yu, T. Suzuki, N. Djourelov, K. Kondo, Y. Ito, V.P. Shantarovich, Chem. Phys. 313, 63 (2005). 